\title{
MMSE precoding for multiuser MISO downlink transmission with non-homogeneous user SNR conditions
}

\author{
Duy HN Nguyen ${ }^{1,2^{*}}$ and Tho Le-Ngoc ${ }^{1}$
}

\begin{abstract}
This paper is concerned with linear precoding designs for multiuser downlink transmissions. We consider a multiple-input single-output (MISO) system with multiple single-antenna user equipment (UE) experiencing nonhomogeneous average signal-to-noise ratio (SNR) conditions. The first part of this work examines different precoding schemes with perfect channel state information (CSI) and average SNR at the base-station (eNB). We then propose a weighted minimum mean squared error (WMMSE) precoder, which takes advantage of the non-homogeneous SNR conditions. Given in a closed-form solution, the proposed WMMSE precoder outperforms other well-known linear precoders, such as zero-forcing (ZF), regularized ZF (RZF), while achieving a close performance to the locally optimal iterative WMMSE (IWMMSE) precoder, in terms of the achievable network sum-rate. In the second part of this work, we consider the non-homogeneous multiuser system with limited and quantized channel quality indicator (CQI) and channel direction indicator (CDI) feedbacks. Based on the CQI and CDI feedback models proposed for the Long-Term Evolution Advanced standard, we then propose a robust WMMSE precoder in a closed-form solution which takes into account the quantization errors. Simulation shows a significant improvement in the achievable network sum-rate by the proposed robust WMMSE precoder, compared to non-robust linear precoder designs.
\end{abstract}

Keywords: MMSE; Multiuser; Precoding; Beamforming; Zero-forcing; Regularized zero-forcing; Quantized feedback; Convex optimization

\section{Introduction}

Multiuser downlink precoding in a wireless system refers to a scenario where a multiple-antenna base station (eNB) multiplexes several user data streams in space, then simultaneously transmits to multiple-user equipment (UE). When full channel state information (CSI) is available at the eNB, it is possible to improve the system's spectral efficiency, albeit proper precoding techniques at the eNB. Research on multiuser precoding designs is plentiful in the literature. Dirty-paper coding (DPC) has been proved as the capacity-achieving multiuser precoding strategy [1-4]. Unfortunately, due to its high complexity implementation that involves random nonlinear coding, DPC only remains

*Correspondence: huu.n.nguyen@mail.mcgill.ca

1 Department of Electrical and Computer Engineering, McGill University, 3480 University Street, H3A 0E9, Montréal, Québec, Canada

${ }^{2}$ INRS-EMT, Université du Québec, 800 de la Gauchetière West, H5A 1K6, Montréal, Québec, Canada as a theoretical benchmark. This makes linear beamforming techniques an attractive alternative because of their reduced complexity relative to DPC.

Finding the optimal transmit beamforming design to maximize the network sum-rate subject to a transmit power constraint is a non-convex and non-trivial problem [5]. Thus, it is desirable to study suboptimal beamforming strategies whose performance is close to that of DPC. In zero-forcing (ZF) precoding, the beamforming weights are designed to eliminate all inter-user interference. While ZF precoding is simple to implement, its performance is poor, especially at low signal-to-noise ratio region [6]. Regularized ZF precoding [6] was then proposed to address the drawback of ZF precoding and improve its performance significantly. Interestingly, regularized ZF precoding can also be interpreted as a Wiener transmit filter, i.e., minimum mean squared error (MMSE) precoding, which is obtained from the minimization of the non-weighted sum mean squared error (MSE) $[7,8]$.

\section{Springer}

(c) 2014 Nguyen and Le-Ngoc; licensee Springer. This is an Open Access article distributed under the terms of the Creative Commons Attribution License (http://creativecommons.org/licenses/by/4.0), which permits unrestricted use, distribution, and reproduction in any medium, provided the original work is properly credited. 
Another approach to find the optimal transmit beamforming vectors is to directly solve the non-convex sumrate problem $[5,9]$. In particular, the work in [9] proposed an iterative algorithm based on the uplink-downlink MSE duality. Using the MSE duality, the algorithm's approach is to iterate between the optimization of the uplink power using geometric programming, uplink receive filters, and downlink receive filters. Different from [9], the approach in [5] was to establish the equivalence between the sum-rate maximization problem and the minimization of weighted sum MSE problem. Thus, the non-convex former problem can be solved via the latter by iteratively updating the weights, the receive filters, and the transmit filters. While both approaches in [5,9] are capable of achieving at least a local optimum, they come with the drawback of high complexity due to the iterative optimization procedure.

In this work, we are interested in designing linear MMSE precoders for a multiple-input single-out (MISO) system with non-homogeneous average signal-to-noise ratio (SNR) conditions. In particular, the UEs, each equipped with a single antenna, are assumed to experience different channel strength statistics and the background noise power, which reflects in different average SNRs. The reason for our consideration to a non-homogeneous system arises from practice where the UEs are randomly located within the eNB's cell limit. Since the large-scale fading depends on the transmission environment and the distance between the eNB and each individual UE, the channel strength statistics to distinct UEs are effectively different. Similarly, the background noise including both thermal noise and co-channel interference from nearby cells, should be also different for each UE.

In the first part of this work, we study the MMSE precoder for the system with perfect CSI and average SNR knowledge at the eNB. Due to the consideration of nonhomogeneous SNR conditions, the approach in [6] to derive the regularized ZF precoder is no longer possible. On the other hand, the MMSE precoder obtained from the non-weighted sum-MSE minimization [7,8] may result in poor network sum-rate performance. Inspired by the iterative weighted minimization of MSE algorithm [5], we propose a weighted MMSE (WMMSE) precoder where the weights and receive coefficients are predetermined. The key aspect of the proposed WMMSE precoder is that it does take advantage of the non-homogeneous average SNR conditions at the UEs to judiciously determine the weights and receive coefficients. The proposed WMMSE precoder will be presented in a closed-form solution using a non-iterative procedure, which is much simpler to obtain than the iterative WMMSE precoder in [5]. Interestingly, numerical results show that the proposed precoder can achieve the sum-rate performance close to the locally optimal one obtained by the iterative algorithm in [5].

In the second part of this work, we examine the MMSE precoding design for the system with limited and quantized channel quality indicator (CQI) and channel direction indicator (CDI) feedbacks. Under this consideration, the eNB only acquires imperfect CSI and average SNR through the quantized CDI and CQI feedbacks from each UE. Thus, robust precoding designs are necessary to address the errors in the quantization process. Robust regularized ZF precoding and robust MMSE precoding have been investigated in $[10,11]$ for the system with homogeneous SNR conditions. Applying the same method to predetermine the weights and receive coefficients, we then propose a robust WMMSE precoder in a closedform solution that accommodates the non-homogeneous SNR conditions as well as the quantization errors. Simulations show that the proposed scheme outperforms the robust MMSE precoder proposed in [11] as well as other non-robust precoding designs.

Notations: $(\mathbf{X})^{*},(\mathbf{X})^{T}$, and $(\mathbf{X})^{H}$ denote the conjugate, transpose, and conjugate transpose (Hermitian operator) of the matrix $\mathbf{X}$, respectively; $[\mathbf{X}]_{u, p}$ stands for the $(u, p)$ th entry of the matrix $\mathbf{X} ; \operatorname{Tr}\{\mathbf{X}\}$ denotes the trace of the matrix $\mathbf{X}$; and $x^{\star}$ denotes the optimal value of the variable $x$.

\section{System model}

Consider the downlink transmission of a multiuser MISO (MU-MISO) system with an $M$-antenna eNB sending independent data symbols to $K$ remote single-antenna UEs. Let $u_{i}$ be the information symbol of UE- $i$ with unit average energy, i.e., $\mathbb{E}\left[\left|u_{i}\right|^{2}\right]=1$, and $\tilde{\mathbf{w}}_{i}$ be the $M \times 1$ beamforming vector for UE- $i$. By means of linear precoding, the transmitted signal is formed as $\mathbf{x}=$ $\sum_{i=1}^{K} \tilde{\mathbf{w}}_{i} u_{i}$, where $\mathbf{x} \in \mathbb{C}^{M}$ is the signal vector transmitted over the $M$ antennas. Given $P$ as the power budget at the eNB, the beamformers are imposed by the power constraint $\sum_{i=1}^{K}\left\|\tilde{\mathbf{w}}_{i}\right\|^{2}=\operatorname{Tr}\left\{\tilde{\mathbf{W}} \tilde{\mathbf{W}}^{H}\right\} \leq P$, where $\tilde{\mathbf{W}}=$ $\left[\tilde{\mathbf{w}}_{1}, \ldots, \tilde{\mathbf{w}}_{K}\right]$.

The signal observed at UE- $i$ can be modeled by

$$
\begin{aligned}
y_{i} & =\sqrt{\rho_{i}} \mathbf{h}_{i}^{H} \mathbf{x}+n_{i} \\
& =\sqrt{\rho_{i}} \mathbf{h}_{i}^{H} \tilde{\mathbf{w}}_{i} u_{i}+\sqrt{\rho_{i}} \mathbf{h}_{i}^{H} \sum_{j \neq i}^{K} \tilde{\mathbf{w}}_{j} u_{j}+n_{i},
\end{aligned}
$$

where $\mathbf{h}_{i}^{*} \in \mathbb{C}^{M \times 1}$ is the small-scale fading channel vector from the eNB to the $i$ th UE, $n_{i}$ is the AWGN at UE- $i$, and $\rho_{i}$ represents the average SNR of UE- $i$. Herein, $\mathbf{h}_{i}$ and $n_{i}$ can be normalized such that they contain i.i.d. circular symmetric complex Gaussian random variables with zero mean and unit variance. Due to the normalization, $\rho_{i}$ 
contains the effects of both large-scale fading and background noise at UE- $i$. To reflect the non-homogeneous SNR conditions, $\rho_{i}$ 's are assumed to be different for the $K$ users. In addition, it is assumed that each UE, say UE- $i$, has perfect estimation of its downlink channel $\mathbf{h}_{i}$ and its average SNR $\rho_{i}$, which are then fed back to the eNB.

Denote $\mathbf{H}=\left[\mathbf{h}_{1}, \ldots, \mathbf{h}_{K}\right]^{H}, \mathbf{u}=\left[u_{1}, \ldots, u_{K}\right]^{T}$, and $\mathbf{n}=$ $\left[n_{1}, \ldots, n_{K}\right]^{T}$. By stacking the received signals at the $K$ UEs into $\mathbf{y}=\left[y_{1}, \ldots, y_{K}\right]^{T}$, one has

$$
\mathbf{y}=\mathbf{\Sigma}^{\frac{1}{2}} \mathbf{H} \tilde{\mathbf{W}} \mathbf{u}+\mathbf{n},
$$

where $\boldsymbol{\Sigma}=\operatorname{diag}\left(\rho_{1}, \ldots, \rho_{K}\right)$.

For a given beamforming matrix $\tilde{\mathbf{W}}$, from Equation 1, the instantaneous signal-to-interference-plus-noise ratio (SINR) at the $i$ th UE can be expressed as

$$
\operatorname{SINR}_{i}=\frac{\rho_{i}\left|\mathbf{h}_{i}^{H} \tilde{\mathbf{w}}_{i}\right|^{2}}{\sum_{j \neq i}^{K} \rho_{i}\left|\mathbf{h}_{i}^{H} \tilde{\mathbf{w}}_{j}\right|^{2}+1} .
$$

The system sum-rate is given by $\sum_{i=1}^{K} \log \left(1+\mathrm{SINR}_{i}\right)$. Thus, subject to the sum power budget $P$ at the eNB, the system sum-rate is maximized by the following optimization

$$
\begin{aligned}
& \underset{\tilde{\mathbf{w}}_{1}, \ldots, \tilde{\mathbf{w}}_{K}}{\operatorname{maximize}} \sum_{i=1}^{K} \log \left(1+\frac{\rho_{i}\left|\mathbf{h}_{i}^{H} \tilde{\mathbf{w}}_{i}\right|^{2}}{\sum_{j \neq i}^{K} \rho_{i}\left|\mathbf{h}_{i}^{H} \tilde{\mathbf{w}}_{j}\right|^{2}+1}\right) \\
& \text { subject to } \sum_{i=1}^{K}\left\|\tilde{\mathbf{w}}_{i}\right\|^{2} \leq P .
\end{aligned}
$$

\section{Review of linear precoding strategies for the multiuser MISO downlink transmission}

It is well-known that the optimization problem (4) is nonconvex due to the presence of the variables $\tilde{\mathbf{w}}_{1}, \ldots, \tilde{\mathbf{w}}_{K}$ in the denominator of the SINR expression (3). Thus, determining the optimal $\tilde{\mathbf{w}}_{i}$ 's is difficult and computationally complicated in practice. In this section, we briefly review three exemplary linear beamforming schemes that may provide good approximated solution to problem (4).

\subsection{Zero-forcing precoding}

In ZF precoding, the beamforming vectors are designed such that they do not induce any inter-user interference, i.e., $\mathbf{h}_{i}^{H} \tilde{\mathbf{w}}_{j}=0$ for $i \neq j$. Thus, ZF precoding is only applicable to the system with $K \leq M$. With ZF precoding, the beamformer vector $\tilde{\mathbf{w}}_{i}$ for UE- $i$ must be scalar to the unnormalized beamformer vector $\mathbf{w}_{i}$, where $\mathbf{W}_{\mathrm{ZF}}=$ $\left[\mathbf{w}_{1}, \ldots, \mathbf{w}_{K}\right]$ is given by

$$
\mathbf{W}_{\mathrm{ZF}}=\mathbf{H}^{H}\left(\mathbf{H H}^{H}\right)^{-1} .
$$

\subsubsection{Equal power scaling allocation}

Under this power allocation strategy, each beamformer vector $\tilde{\mathbf{w}}_{i}$ is scaled up from the unnormalized beamformer vector $\mathbf{w}_{i}$ with the same normalization factor $\eta$, i.e., $\tilde{\mathbf{w}}_{i}=$ $\eta \mathbf{w}_{i}$. To meet the sum power constraint at the eNB, $\eta$ is given by $\eta=\sqrt{P / \operatorname{Tr}\left\{\mathbf{W}_{\mathrm{ZF}} \mathbf{W}_{\mathrm{ZF}}^{H}\right\}}$. Hereafter, this ZF precoding and equal power scaling allocation strategy will be referred to as the ZF-EPS scheme.

\subsubsection{Optimal power allocation - water-filling solution}

Under the optimal power allocation strategy, each beamformer vector $\tilde{\mathbf{w}}_{i}$ is scaled up from the unnormalized beamformer vector $\mathbf{w}_{i}$ with a distinct factor $\eta_{i} \triangleq \sqrt{p_{i}}$, which is optimally determined. Replacing $\tilde{\mathbf{w}}_{i}=\sqrt{p_{i}} \mathbf{w}_{i}$, the optimization (4) can be restated as

$$
\begin{gathered}
\underset{p_{1}, \ldots, p_{K}}{\operatorname{maximize}} \sum_{i=1}^{K} \log \left(1+\rho_{i} p_{i}\right) \\
\text { subject to } \\
\sum_{i=1}^{K} \gamma_{i} p_{i} \leq P \\
p_{i} \geq 0,
\end{gathered}
$$

where $\gamma_{i}=\left[\left(\mathbf{H H}^{H}\right)^{-1}\right]_{i, i}$. The optimal solution to the above problem can be easily found by the water-filling solution

$$
p_{i}=\left[\frac{\mu}{\gamma_{i}}-\frac{1}{\rho_{i}}\right]^{+}, \forall i,
$$

where $[x]^{+}=\max (x, 0)$ and the water level $\mu$ is chosen to meet the sum power constraint $\sum_{i=1}^{K}\left[\mu-\gamma_{i} \rho_{i}^{-1}\right]^{+}=P$. In general, a user with higher SNR will be allocated with more power in the water-filling process. Hereafter, the ZF precoding with the water-filling power strategy will be referred to as the ZF-WF scheme. Certainly, the ZF-WF scheme always outperforms the ZF-EPS scheme.

\subsection{Regularized zero-forcing precoding}

Due to the ill-conditioned behavior of the largest eigenvalue of $\left(\mathbf{H H}^{H}\right)^{-1}$, the sum-rate obtained by the ZF-EPS stays constant as $M=K \rightarrow \infty$ [6]. To address to the poor performance of the ZF-EPS scheme, the work in [6] proposed the regularized ZF precoding where the unnormalized beamformer matrix $\mathbf{W}_{\text {RZF }}$ is given by

$$
\mathbf{W}_{\text {RZF }}=\mathbf{H}^{H}\left(\mathbf{H H}^{H}+\alpha \mathbf{I}\right)^{-1},
$$

where $\alpha>0$ is the regularizer factor. The normalized beamformer matrix is then given as $\tilde{\mathbf{W}}=\eta \mathbf{W}_{\mathrm{RZF}}$, where $\eta$ is given as $\eta=\sqrt{P / \operatorname{Tr}\left\{\mathbf{W}_{\mathrm{RZF}} \mathbf{W}_{\mathrm{RZF}}^{H}\right\}}$.

By maximizing the SINR at the UEs, the optimal regularizer was derived as $\alpha^{\star}=K / P$ [6]. Note that this optimal regularizer was only obtained for the case of homogeneous SNR conditions $\rho_{i}=1, \forall i$. In contrast, for the non-homogeneous case, obtaining a closed-form statistic on the achievable SINR at the UEs is not possible. Thus, 
the technique utilized in [6] is no longer applicable. Fortunately, the regularized ZF precoding can also be obtained as the MMSE transmit filter through the non-weighted sum-MSE minimization $[7,8]$

$$
\begin{aligned}
& \underset{\mathbf{W}_{\mathrm{RZF}}, \eta}{\operatorname{minimize}} \mathbb{E}\left\{\left\|\mathbf{u}-\eta^{-1} \Sigma^{-\frac{1}{2}} \mathbf{y}\right\|^{2}\right\} \\
& \text { subject to } \eta^{2} \operatorname{Tr}\left\{\mathbf{W}_{\mathrm{RZF}} \mathbf{W}_{\mathrm{RZF}}^{H}\right\} \leq P,
\end{aligned}
$$

where $\operatorname{MSE}_{i}=\mathbb{E}\left\{\left|u_{i}-\eta^{-1} \rho_{i}^{-1 / 2} y_{i}\right|^{2}\right\}$ is the MSE at UE- $i .^{\text {a }}$

Following the optimization procedure in [7], the optimal solution to problem (8) can be derived as

$$
\mathbf{W}_{\mathrm{RZF}}=\mathbf{H}^{H}\left(\mathbf{H H}^{H}+\frac{\sum_{i=1}^{K}\left(1 / \rho_{i}\right)}{P} \mathbf{I}\right)^{-1} .
$$

Again, the normalized beamformer matrix is then given by $\tilde{\mathbf{W}}=\eta \mathbf{W}_{\text {RZF }}$. Hereafter, the precoder given in (9) will be referred to as the regularized ZF (RZF) precoder.

\subsection{Iterative weighted minimization of mean squared error algorithm}

In [5], the authors proposed a numerical method to solve the non-convex optimization problem (4) by considering an equivalent minimization of weighted MSE problem

$$
\begin{aligned}
& \underset{\Lambda, \Omega, \tilde{\mathbf{W}}}{\operatorname{minimize}} \mathbb{E}\left\{\left\|\Omega^{\frac{1}{2}}(\mathbf{u}-\Lambda \mathbf{y})\right\|^{2}\right\}-\log \operatorname{det} \Omega \\
& \text { subject to } \operatorname{Tr}\left\{\tilde{\mathbf{W}} \tilde{\mathbf{W}}^{H}\right\} \leq P,
\end{aligned}
$$

where $\Lambda=\operatorname{diag}\left(\lambda_{1}, \ldots, \lambda_{K}\right)$ is the set of receive coefficients and $\Omega=\operatorname{diag}\left(\omega_{1}, \ldots, \omega_{K}\right)$ is the set of weights associated with the MSEs at the $K$ UEs. Note that the receive coefficients and the weights are to be optimized as well as the beamforming matrix $\tilde{\mathbf{W}}$ in problem (10). Compared to the non-weighted MMSE problem (8), the optimization in (10) assigns distinct receive coefficients $\lambda_{i}$ 's as well as distinct weights $\omega_{i}$ 's to the achievable MSEs $e_{i} \triangleq \mathbb{E}\left\{\left|u_{i}-\lambda_{i} y_{i}\right|^{2}\right\}$ 's for different UEs.

Although problem (10) is not jointly convex, it is convex over each set of variables $\tilde{\mathbf{W}}, \Omega$, and $\Lambda$ [5]. Thus, it is possible to solve problem (10) by alternately optimizing over one set of variables while keeping the other two fixed. More specifically, by fixing $\tilde{\mathbf{W}}$ and $\omega_{i}$ 's, the optimal receive coefficient $\lambda_{i}$ to minimize the MSE is the MMSE receiver

$$
\begin{aligned}
\lambda_{i} & =\arg \min _{\lambda_{i}} \mathbb{E}\left\{\left|u_{i}-\lambda_{i} y_{i}\right|^{2}\right\} \\
& =\left(\sum_{j=1}^{K} \rho_{i}\left|\mathbf{h}_{i}^{H} \tilde{\mathbf{w}}_{j}\right|^{2}+1\right)^{-1} \sqrt{\rho_{i}} \tilde{\mathbf{w}}_{i}^{H} \mathbf{h}_{i} .
\end{aligned}
$$

Fixing $\tilde{\mathbf{W}}$ and $\lambda_{i}$ 's, the optimal weight $\omega_{i}$ can be determined by the unconstrained optimization

$$
\begin{aligned}
\omega_{i} & =\arg \min _{\omega_{i}} \omega_{i} e_{i}-\log \omega_{i} \\
& =e_{i}^{-1}=1+\operatorname{SINR}_{i}=\frac{\sum_{j=1}^{K} \rho_{i}\left|\mathbf{h}_{i}^{H} \tilde{\mathbf{w}}_{j}\right|^{2}+1}{\sum_{j \neq i}^{K} \rho_{i}\left|\mathbf{h}_{i}^{H} \tilde{\mathbf{w}}_{j}\right|^{2}+1} .
\end{aligned}
$$

Finally, fixing $\omega_{i}^{\prime}$ 's and $\lambda_{i}$ 's, the optimal beamformer vector $\tilde{\mathbf{w}}_{i}$ is given by

$$
\begin{aligned}
\tilde{\mathbf{w}}_{i} & =\arg \min _{\tilde{\mathbf{w}}_{i}} \sum_{j=1}^{K} \omega_{j} \mathbb{E}\left\{\left|u_{j}-\lambda_{j} y_{j}\right|^{2}\right\} \\
& =\left(\sum_{j=1}^{K}\left|\lambda_{j}\right|^{2} \rho_{j} \omega_{j} \mathbf{h}_{j} \mathbf{h}_{j}^{H}+\mu \mathbf{I}\right)^{-1} \mathbf{h}_{i} \lambda_{i}^{*} \omega_{i} \sqrt{\rho_{i}},
\end{aligned}
$$

where $\mu$ is adjusted to meet the power constraint in (10). The transmit beamforming set $\tilde{\mathbf{w}}_{i}$ 's can be put together into a matrix form as

$$
\begin{aligned}
\tilde{\mathbf{W}} & =\left(\mathbf{H}^{H} \Lambda^{H} \Omega \Sigma \Lambda \mathbf{H}+\mu \mathbf{I}\right)^{-1} \mathbf{H}^{H} \Lambda^{H} \Omega \Sigma^{\frac{1}{2}} \\
& =\mathbf{H}^{H}\left[\mathbf{H} \mathbf{H}^{H}+\mu\left(\Lambda^{H} \Omega \Sigma \Lambda\right)^{-1}\right]^{-1} \Lambda^{-1} \Sigma^{-\frac{1}{2}},
\end{aligned}
$$

where the matrix manipulation $\mathbf{A}^{-1} \mathbf{B}\left(\mathbf{B}^{T} \mathbf{A}^{-1} \mathbf{B}+\mathbf{I}\right)^{-1}=$ $\left(\mathbf{B B}^{T}+\mathbf{A}\right)^{-1} \mathbf{B}$ is taken (cf. Equation 162 in [12]).

As proved in [5], the alternate optimization procedure of $\lambda_{i}{ }^{\prime}$ s, $\omega_{i}$ 's, and $\tilde{\mathbf{W}}$ converges monotonically to at least a local optimal solution. The algorithm, will be referred to as the iterative minimization of the weighted MSE algorithm (IWMMSE), can be summarized as follows:

1. Initialize: randomize $\tilde{\mathbf{w}}_{i}, \forall i$ with $\left\|\tilde{\mathbf{w}}_{i}\right\|^{2}=P / K$.

2. Repeat until convergence:

- Update the receive coefficients $\lambda_{i}$ 's as in (11).

- Update the weights $\omega_{i}^{\prime}$ 's as in (12).

- Update the transmit beamformer $\tilde{\mathbf{W}}$ (14).

\section{The proposed WMMSE precoder with perfect CSI and average SNR knowledge}

\subsection{The proposed WMMSE precoder}

As reviewed in Section 3, the IWMMSE algorithm can obtain at least a locally optimal solution to the original sum-rate maximization problem (4). While the IWMMSE algorithm can significantly outperform the ZF-EPS, ZFWF, and RZF schemes, it comes with the drawback of high complexity due to the iterative optimization procedure. In this section, we propose a WMMSE precoder in a closed-form solution, which can approach the performance of the IWMMSE scheme. The key aspect of the proposed WMMSE precoder is to take advantage of the 
non-homogeneous SNR conditions at the UE to predetermine the weights and receive coefficients and thus remove the iterative procedure of the IWMMSE algorithm.

Let $g_{i}=\left\|\mathbf{h}_{i}\right\| / \sigma_{n_{i}}$ denote the effective small-scale channel gain to UE- $i$, one has

$$
\mathbf{H}=\mathbf{G}\left[\tilde{\mathbf{h}}_{1}, \ldots, \tilde{\mathbf{h}}_{K}\right]^{H}=\mathbf{G} \tilde{\mathbf{H}},
$$

where $\mathbf{G}=\operatorname{diag}\left(g_{1}, \ldots, g_{K}\right)$ and $\tilde{\mathbf{h}}_{i}=\mathbf{h}_{i} / g_{i}$ is the unit norm channel vector to UE- $i$. Let us define the weighted MSE cost function

$$
\mathrm{MSE}=\mathbb{E}\left\{\left\|\mathbf{G} \boldsymbol{\Sigma}^{\frac{1}{2}}\left(\mathbf{u}-\eta^{-1} \mathbf{G}^{-1} \boldsymbol{\Sigma}^{-\frac{1}{2}} \mathbf{y}\right)\right\|^{2}\right\},
$$

where $\eta$ is normalization factor to meet the power constraint at the eNB. Herein, $\eta^{-1} g_{i}^{-1} \rho_{i}^{-1 / 2}$ is chosen the receive coefficient, whereas $g_{i}^{2} \rho_{i}$ is chosen as the weight for the MSE at UE- $i$, which is given as $\mathbb{E}\left\{\left|u_{i}-\eta^{-1} g_{i}^{-1} \rho_{i}^{-1 / 2} y_{i}\right|^{2}\right\}$. The intuitions for choosing these coefficients are as follows:

- The receive coefficients $\eta^{-1} g_{i}^{-1} \rho_{i}^{-1 / 2}$, s reflect the different SNR conditions. In addition, these coefficients can normalize the channels $\sqrt{\rho_{i}} \mathbf{h}_{i}$ 's to the same statistics with the same unit norm.

- The weights $g_{i}^{2} \rho_{i}^{\prime}$ 's normalize (or whiten) the colored

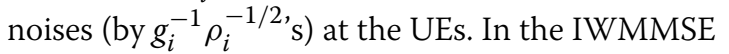
algorithm, the optimal weight $\omega_{i}$, given by $1+\operatorname{SINR}_{i}$ as in (12), is analogous to the instantaneous SINR at the UE- $i$. Our predetermined weight $g_{i}^{2} \rho_{i}$ indeed reflects this instantaneous SINR, especially at high-SNR region.

It is to be noted that the proposed approach in minimizing the weighted MSE maintains higher emphasis for users with better SNR conditions. Unlike the non-weighted MSE minimization in (8), which indirectly leads to balance the bit-error-rate performance across the users, our approach is to boost the sum-rate performance instead.

Denote $\gamma=\eta^{-2}$ and let us consider the following weighted MSE minimization problem

$$
\begin{aligned}
& \underset{\mathbf{W}, \gamma}{\operatorname{minimize}} \mathbb{E}\left\{\left\|\boldsymbol{\Sigma}^{\frac{1}{2}} \mathbf{G}\left(\mathbf{u}-\sqrt{\gamma} \mathbf{G}^{-1} \boldsymbol{\Sigma}^{-\frac{1}{2}} \mathbf{y}\right)\right\|^{2}\right\} \\
& \text { subject to } \operatorname{Tr}\left\{\mathbf{W} \mathbf{W}^{H}\right\} \leq \gamma P .
\end{aligned}
$$

Expanding the cost function, one has

$$
\begin{aligned}
\mathrm{MSE} & =\mathbb{E}\left\{\left\|\boldsymbol{\Sigma}^{\frac{1}{2}} \mathbf{G}(\mathbf{I}-\tilde{\mathbf{H}} \mathbf{W}) \mathbf{u}-\sqrt{\gamma} \mathbf{n}\right\|^{2}\right\} \\
& =\operatorname{Tr}\left\{\Sigma^{\frac{1}{2}} \mathbf{G}(\mathbf{I}-\tilde{\mathbf{H}} \mathbf{W})(\mathbf{I}-\tilde{\mathbf{H}} \mathbf{W})^{H} \mathbf{G} \Sigma^{\frac{1}{2}}\right\}+\gamma K .
\end{aligned}
$$

It is observed that both the cost function and constraints are quadratic in $\mathbf{W}$ and linear in $\gamma$. Thus, problem (17) is jointly convex in $\mathbf{W}$ and $\gamma$, which enables efficiently optimization techniques to find its optimal solution [13].

Let $\mu$ denote the Lagrangian multiplier associated with the power constraint, the Lagrangian of the convex problem (17) is then given by

$$
\begin{aligned}
\mathcal{L}(\mathbf{W}, \gamma, \mu)= & \operatorname{Tr}\left\{\mathbf{W}^{H} \tilde{\mathbf{H}}^{H} \boldsymbol{\Sigma} \mathbf{G}^{2} \tilde{\mathbf{H}} \mathbf{W}\right\} \\
& -\operatorname{Tr}\left\{\boldsymbol{\Sigma} \mathbf{G}^{2} \tilde{\mathbf{H}} \mathbf{W}+\mathbf{W}^{H} \tilde{\mathbf{H}}^{H} \mathbf{G}^{2} \boldsymbol{\Sigma}\right\} \\
& +\operatorname{Tr}\left\{\boldsymbol{\Sigma} \mathbf{G}^{2}\right\}+\gamma K+\mu\left(\operatorname{Tr}\left\{\mathbf{W} \mathbf{W}^{H}\right\}-\gamma P\right) \\
= & \operatorname{Tr}\left\{\mathbf{W}^{H}\left(\tilde{\mathbf{H}}^{H} \boldsymbol{\Sigma} \mathbf{G}^{2} \tilde{\mathbf{H}}+\mu \mathbf{I}\right) \mathbf{W}\right\} \\
& -\operatorname{Tr}\left\{\boldsymbol{\Sigma} \mathbf{G}^{2} \tilde{\mathbf{H}} \mathbf{W}+\mathbf{W}^{H} \tilde{\mathbf{H}}^{H} \mathbf{G}^{2} \boldsymbol{\Sigma}\right\} \\
& +\gamma K-\mu \gamma P+\operatorname{Tr}\left\{\mathbf{\Sigma} \mathbf{G}^{2}\right\} .
\end{aligned}
$$

Since the gradient of the Lagrangian vanishes at optimality [13], one has

$$
\left.\frac{\partial \mathcal{L}(\mathbf{W}, \gamma, \mu)}{\partial \gamma}\right|_{\gamma=\gamma^{\star}, \mu=\mu^{\star}}=K-\mu^{\star} P=0 .
$$

Thus, the optimal dual variable $\mu^{\star}$ is given by $\mu^{\star}=\frac{K}{P}$. In addition,

$$
\begin{gathered}
\left.\frac{\partial \mathcal{L}(\mathbf{W}, \gamma, \mu)}{\partial \mathbf{W}^{*}}\right|_{\mathbf{W}=\mathbf{W}^{\star}, \mu=\mu^{\star}}=2\left(\tilde{\mathbf{H}}^{H} \boldsymbol{\Sigma} \mathbf{G}^{2} \tilde{\mathbf{H}}+\mu^{\star} \mathbf{I}\right) \mathbf{W}^{\star} \\
-2 \tilde{\mathbf{H}}^{H} \mathbf{G}^{2} \boldsymbol{\Sigma}=\mathbf{0} .
\end{gathered}
$$

Subsequently, the optimal unnormalized beamforming matrix $\mathbf{W}^{\star}$ can be obtained in a closed-form solution

$$
\begin{aligned}
\mathbf{W}^{\star} & =\left(\tilde{\mathbf{H}}^{H} \boldsymbol{\Sigma} \mathbf{G}^{2} \tilde{\mathbf{H}}+\frac{K}{P} \mathbf{I}\right)^{-1} \tilde{\mathbf{H}}^{H} \mathbf{G}^{2} \boldsymbol{\Sigma} \\
& =\tilde{\mathbf{H}}^{H}\left(\tilde{\mathbf{H}} \tilde{\mathbf{H}}^{H}+\frac{K}{P} \boldsymbol{\Sigma}^{-1} \mathbf{G}^{-2}\right)^{-1} \\
& =\mathbf{H}^{H}\left(\mathbf{H} \mathbf{H}^{H}+\frac{K}{P} \boldsymbol{\Sigma}^{-1}\right)^{-1} \mathbf{G},
\end{aligned}
$$

where the matrix manipulation $\mathbf{A}^{-1} \mathbf{B}\left(\mathbf{B}^{T} \mathbf{A}^{-1} \mathbf{B}+\mathbf{I}\right)^{-1}=$ $\left(\mathbf{B B}^{T}+\mathbf{A}\right)^{-1} \mathbf{B}$ is again utilized. Finally, the optimal normalization factor $\eta^{\star}$ is given by $\eta^{\star}=\sqrt{P / \operatorname{Tr}\left\{\mathbf{W}^{\star}\left(\mathbf{W}^{\star}\right)^{H}\right\}}$ and the optimal normalized beamforming matrix is $\tilde{\mathbf{W}}^{\star}=$ $\eta^{\star} \mathbf{W}^{\star}$. Hereafter, the proposed precoder in (22) will be referred to as the WMMSE precoder.

Remark 1. It is observed that the proposed WMMSE precoder resembles the one obtained from the IWMMSE algorithm in (14). Compared with the RZF precoder (9), it is interesting to observe that the precoder (22) has a non-identity regularizer matrix in $\Sigma^{-1}$. 


\section{MMSE precoding with quantized CDI and CQI feedbacks}

\subsection{CDI and CQI feedback model}

This section considers the multiuser MIMO system where the eNB only possesses imperfect channel and SNR estimation through the limited quantized CDI and CQI feedbacks from the UEs. No knowledge on the average SNR, $\rho_{i}$, is available at the eNB. Each UE, say UE- $i$, has perfect knowledge of $\mathbf{h}_{i}$, can quantize the direction of its channel $\tilde{\mathbf{h}}_{i}=\mathbf{h}_{i} /\left\|\mathbf{h}_{i}\right\|$ to a unit norm vector $\hat{\mathbf{h}}_{i}$. Let $B$ denote the number of CDI feedback bits from each UE, and $\mathcal{C}_{i}$ denote the codebook for UE- $i$. We assume that the $\operatorname{codebook} \mathcal{C}_{i}=$ $\left\{\mathbf{c}_{i 1}, \ldots, \mathbf{c}_{i 2^{B}}\right\}$, consisting of $2^{B} M$-dimensional randomized vectors with unit norm, is predesigned and known at the eNB and the corresponding UE- $i$. It is further assumed that each codebook is designed independently of each other. At UE- $i$, the quantized vector $\hat{\mathbf{h}}_{i}$ is chosen by the criterion

$$
\hat{\mathbf{h}}_{i}=\arg \max _{\mathbf{c}_{i n} \in \mathcal{C}_{i}}\left|\tilde{\mathbf{h}}_{i}^{H} \mathbf{c}_{i n}\right| .
$$

UE- $i$ then feeds back only the index of $\hat{\mathbf{h}}_{i}$ to the eNB, which requires $B$ bits per feedback.

Besides the CDI feedback, each UE may need to feed back its CQI to inform the eNB its effective channel condition. Typically, the CQI is based on the instantaneous SNR at the UE $[14]^{\mathrm{b}}$

$$
\mathrm{SNR}_{i}=(P / M) \rho_{i}\left\|\mathbf{h}_{i}\right\|^{2}=(P / M) g_{i}^{2} \rho_{i}
$$

Instead of transmitting the average SNR $\rho_{i}$ or the channel gain $g_{i}^{2}$ exactly, UE- $i$ uses CQI feedback to inform the eNB its instantaneous SNR. With unquantized CQI feedbacks, the eNB can perfectly recover the effective channel gain $g_{i}^{2} \rho_{i}$ for each user. Whereas with quantized CQI feedbacks, the measured SNR at each UE is mapped into a certain number of CQI bits. In LTE-Advanced, the number of CQI feedback bits is typically set at 4 [15]. From this CQI, the eNB then can apply the CQI-SNR mapping to approximate the SNR for each UE [15]. Denote $\widehat{\mathrm{SNR}}_{i}$ as the quantized SNR value obtained from the CQISNR mapping at UE- $i$, its effective channel gain can be approximated as $\hat{g}_{i}^{2} \hat{\rho}_{i}=(M / P) \widehat{\mathrm{SNR}}_{i}$.

Note that the SINR at UE- $i$ is unchanged by replacing $\mathbf{h}_{i}$ and $\rho_{i}$ by $\tilde{\mathbf{h}}_{i}$ and $g_{i}^{2} \rho_{i}$, respectively. Thus, in case of limited quantized CDI and CQI feedbacks, the linear precoding strategies ZF-EPS, ZF-WF, RZF, IWMMSE mentioned in Section 3 can be straightforwardly applied by replacing the channel $\mathbf{h}_{i}$ 's with the quantized channel $\hat{\mathbf{h}}_{i}$ 's and replacing the average $\mathrm{SNR} \rho_{i}$ 's with the approximated effective channel gain $\hat{g}_{i}^{2} \hat{\rho}_{i}$ 's. However, these designs are non-robust to the quantization errors.

\subsection{The proposed robust MMSE precoding for limited quantized feedbacks}

In this section, we examine the optimal beamforming design in order to minimize the MSE cost function (16) with imperfect CSI and average SNR knowledge obtained from the limited CDI and CQI feedbacks. Let us start with the following decomposition on the normalized channel $\tilde{\mathbf{h}}_{i}$ to UE- $i[16]$

$$
\tilde{\mathbf{h}}_{i}=\sqrt{1-z_{i}} \hat{\mathbf{h}}_{i}+\sqrt{z_{i}} \mathbf{s}_{i},
$$

where $\mathbf{s}_{i}$ is a unit-norm isotropically distributed vector in the null space of $\hat{\mathbf{h}}_{i}$ with zero-mean, i.e., $\mathbb{E}\left[\mathbf{s}_{i}\right]=\mathbf{0}$, and $z_{i}=1-\left|\tilde{\mathbf{h}}_{i}^{H} \hat{\mathbf{h}}_{i}\right|^{2}$ is independent of $\mathbf{s}_{i}$. As given in [16], the expectation of $z_{i}$ is given by

$$
\mathbb{E}\left\{z_{i}\right\}=\frac{M-1}{M} \delta
$$

where $\delta=2^{-B /(M-1)}$. Due to the quantization error, the channel matrix $\mathbf{H}$ can be decomposed as

$$
\mathbf{H}=\mathbf{G} \tilde{\mathbf{H}}=\mathbf{G}(\mathbf{I}-\mathbf{Z})^{\frac{1}{2}} \hat{\mathbf{H}}+\mathbf{G Z}^{\frac{1}{2}} \mathbf{S},
$$

where $\mathbf{Z}=\operatorname{diag}\left(z_{1}, \ldots, z_{K}\right), \hat{\mathbf{H}}=\left[\hat{\mathbf{h}}_{1}, \ldots, \hat{\mathbf{h}}_{K}\right]^{H}$, and $\mathbf{S}=$ $\left[\mathbf{s}_{1}, \ldots, \mathbf{s}_{K}\right]^{H}$. Given the known quantized channel $\hat{\mathbf{H}}$, we attempt to minimize the WMMSE by solving the following optimization

$$
\begin{aligned}
& \underset{\mathbf{W}, \gamma}{\operatorname{minimize}} \mathbb{E}\left\{\left\|\boldsymbol{\Sigma}^{\frac{1}{2}} \mathbf{G}\left(\mathbf{u}-\sqrt{\gamma} \mathbf{G}^{-1} \boldsymbol{\Sigma}^{-\frac{1}{2}} \mathbf{y}\right)\right\|^{2} \mid \hat{\mathbf{H}}\right\} \\
& \text { subject to } \operatorname{Tr}\left\{\mathbf{W} \mathbf{W}^{H}\right\} \leq \gamma P .
\end{aligned}
$$

For now, we assume that the effective channel gains $g_{i}^{2} \rho_{i}$ 's in $\mathbf{G} \boldsymbol{\Sigma}^{\frac{1}{2}}$ are perfectly known at eNB for the purpose of solving this optimization problem. Expanding the cost function in (28), one has

$$
\begin{aligned}
\mathrm{MSE}= & \mathbb{E}\left\{\| \boldsymbol{\Sigma}^{\frac{1}{2}} \mathbf{G}\left(\mathbf{I}-(\mathbf{I}-\mathbf{Z})^{\frac{1}{2}} \hat{\mathbf{H} W}\right) \mathbf{u}\right. \\
& \left.-\boldsymbol{\Sigma}^{\frac{1}{2}} \mathbf{G} \mathbf{Z}^{\frac{1}{2}} \mathbf{S W u}-\sqrt{\gamma} \mathbf{n} \|\left.\right|^{2} \hat{\mathbf{H}}\right\} \\
= & \mathbb{E}\left\{\left\|\boldsymbol{\Sigma}^{\frac{1}{2}} \mathbf{G}\left(\mathbf{I}-(\mathbf{I}-\mathbf{Z})^{\frac{1}{2}} \hat{\mathbf{H} W}\right)\right\|_{F}^{2}\right\} \\
& +\mathbb{E}\left\{\left\|\boldsymbol{\Sigma}^{\frac{1}{2}} \mathbf{G} \mathbf{Z}^{\frac{1}{2}} \mathbf{S W}\right\|_{F}^{2} \mid \hat{\mathbf{H}}\right\}+\gamma K .
\end{aligned}
$$


The first component of the cost function (29) can be decomposed as

$$
\begin{aligned}
\mathbb{E} & \left\{\left\|\boldsymbol{\Sigma}^{\frac{1}{2}} \mathbf{G}\left(\mathbf{I}-(\mathbf{I}-\mathbf{Z})^{\frac{1}{2}} \hat{\mathbf{H}} \mathbf{W}\right)\right\|_{F}^{2}\right\} \\
= & \operatorname{Tr}\left\{\boldsymbol{\Sigma} \mathbf{G}^{2}\right\}+\mathbb{E}_{z}\left\{\operatorname{Tr}\left\{\mathbf{W}^{H} \hat{\mathbf{H}}^{H} \boldsymbol{\Sigma} \mathbf{G}^{2}(\mathbf{I}-\mathbf{Z}) \hat{\mathbf{H}} \mathbf{W}\right\}\right\} \\
& -\mathbb{E}_{z}\left\{\operatorname{Tr}\left\{\boldsymbol{\Sigma} \mathbf{G}^{2}(\mathbf{I}-\mathbf{Z})^{\frac{1}{2}} \hat{\mathbf{H}} \mathbf{W}+\mathbf{W}^{H} \hat{\mathbf{H}}^{H}(\mathbf{I}-\mathbf{Z})^{\frac{1}{2}} \mathbf{G}^{2} \boldsymbol{\Sigma}\right\}\right\} \\
= & \operatorname{Tr}\left\{\boldsymbol{\Sigma} \mathbf{G}^{2}\right\}+\left(1-\frac{M-1}{M} \delta\right) \operatorname{Tr}\left\{\mathbf{W}^{H} \hat{\mathbf{H}}^{H} \boldsymbol{\Sigma} \mathbf{G}^{2} \hat{\mathbf{H}} \mathbf{W}\right\} \\
& -\zeta \operatorname{Tr}\left\{\boldsymbol{\Sigma} \mathbf{G}^{2} \hat{\mathbf{H}} \mathbf{W}+\mathbf{W}^{H} \hat{\mathbf{H}}^{H} \mathbf{G}^{2} \boldsymbol{\Sigma}\right\},
\end{aligned}
$$

where $\zeta=\mathbb{E}\left[\sqrt{1-z_{i}}\right]$. As will be shown later, the exact calculation of $\zeta$ is not needed.

Note that the expectation of $\mathbf{s}_{i}$ is $\mathbf{0}$ and the covariance matrix of $\mathbf{s}_{i}$ is given by (cf. Equation 33 in [11])

$$
\mathbb{E}\left\{\mathbf{s}_{i} \mathbf{s}_{i}^{H}\right\}=\frac{1}{M-1}\left(\mathbf{I}_{M}-\hat{\mathbf{h}}_{i} \hat{\mathbf{h}}_{i}^{H}\right) .
$$

Then, one has (cf. Equation 329 in [12])

$$
\begin{aligned}
\mathbb{E}\left\{\mathbf{s}_{i}^{H} \mathbf{W} \mathbf{W}^{H} \mathbf{s}_{i}\right\} & =\operatorname{Tr}\left\{\mathbf{W}^{H} \operatorname{var}\left[\mathbf{s}_{i}\right] \mathbf{W}\right\}+\mathbb{E}\left\{\mathbf{s}_{i}\right\}^{H} \mathbf{W}^{H} \mathbf{W} \mathbb{E}\left\{\mathbf{s}_{i}\right\} \\
& =\frac{1}{M-1} \operatorname{Tr}\left\{\mathbf{W}^{H}\left(\mathbf{I}-\hat{\mathbf{h}}_{i} \hat{\mathbf{h}}_{i}^{H}\right) \mathbf{W}\right\} .
\end{aligned}
$$

Therefore, the second component of the cost function (29) can be decomposed as

$$
\begin{aligned}
\mathbb{E}\left\{\left\|\boldsymbol{\Sigma}^{\frac{1}{2}} \mathbf{G} \mathbf{Z}^{\frac{1}{2}} \mathbf{S W}\right\|_{F}^{2} \mid \hat{\mathbf{H}}\right\}= & \frac{M-1}{M} \delta \sum_{i=1}^{K} \mathbb{E}\left\{\rho_{i} g_{i}^{2} \mathbf{s}_{i}^{H} \mathbf{W} \mathbf{W}^{H} \mathbf{s}_{i}\right\} \\
= & \frac{\delta}{M}\left(\operatorname{Tr}\left\{\boldsymbol{\Sigma} \mathbf{G}^{2}\right\} \operatorname{Tr}\left\{\mathbf{W}^{H} \mathbf{W}\right\}\right. \\
& \left.-\operatorname{Tr}\left\{\mathbf{W}^{H} \hat{\mathbf{H}}^{H} \boldsymbol{\Sigma} \mathbf{G}^{2} \hat{\mathbf{H}} \mathbf{W}\right\}\right) .
\end{aligned}
$$

Consequently, the cost function (29) can be simplified as

$$
\begin{aligned}
& \text { MSE }= \operatorname{Tr}\left\{\boldsymbol{\Sigma}^{2}\right\}+(1-\delta) \operatorname{Tr}\left\{\mathbf{W}^{H} \hat{\mathbf{H}}^{H} \boldsymbol{\Sigma} \mathbf{G}^{2} \hat{\mathbf{H}} \mathbf{W}\right\} \\
&+\frac{\delta \operatorname{Tr}\left\{\boldsymbol{\Sigma} \mathbf{G}^{2}\right\}}{M} \operatorname{Tr}\left\{\mathbf{W}^{H} \mathbf{W}\right\} \\
&-\zeta \operatorname{Tr}\left\{\boldsymbol{\Sigma} \mathbf{G}^{2} \hat{\mathbf{H}} \mathbf{W}+\mathbf{W}^{H} \hat{\mathbf{H}}^{H} \mathbf{G}^{2} \boldsymbol{\Sigma}\right\}+\gamma K .
\end{aligned}
$$

It is observed that the above cost function is quadratic in $\mathbf{W}$ and linear in $\gamma$. Thus, like problem (17), problem (28) is also convex, which facilitates the finding of its optimal solution. Let $\mu$ denote the Lagrangian multiplier associated with the power constraint, the Lagrangian of the convex problem (28) is then given by

$$
\begin{aligned}
\mathcal{L}(\mathbf{W}, \gamma, \mu)= & \operatorname{Tr}\left\{\boldsymbol{\Sigma} \mathbf{G}^{2}\right\}+(1-\delta) \operatorname{Tr}\left\{\mathbf{W}^{H} \hat{\mathbf{H}}^{H} \boldsymbol{\Sigma} \mathbf{G}^{2} \hat{\mathbf{H}} \mathbf{W}\right\} \\
& +\frac{\delta \operatorname{Tr}\left\{\boldsymbol{\Sigma} \mathbf{G}^{2}\right\}}{M} \operatorname{Tr}\left\{\mathbf{W}^{H} \mathbf{W}\right\} \\
& -\zeta \operatorname{Tr}\left\{\boldsymbol{\Sigma} \mathbf{G}^{2} \hat{\mathbf{H}} \mathbf{W}+\mathbf{W}^{H} \hat{\mathbf{H}}^{H} \mathbf{G}^{2} \boldsymbol{\Sigma}\right\}+\gamma K \\
& +\mu\left(\operatorname{Tr}\left\{\mathbf{W} \mathbf{W}^{H}\right\}-\gamma P\right) \\
= & \operatorname{Tr}\left\{\mathbf{W}^{H}\left((1-\delta) \hat{\mathbf{H}}^{H} \boldsymbol{\Sigma} \mathbf{G}^{2} \hat{\mathbf{H}}+\frac{\delta \operatorname{Tr}\left\{\mathbf{\Sigma} \mathbf{G}^{2}\right\}}{M} \mathbf{I}+\mu \mathbf{I}\right) \mathbf{W}\right\} \\
& -\zeta \operatorname{Tr}\left\{\mathbf{\Sigma} \mathbf{G}^{2} \hat{\mathbf{H}} \mathbf{W}+\mathbf{W}^{H} \hat{\mathbf{H}}^{H} \mathbf{G}^{2} \mathbf{\Sigma}\right\}+\gamma K-\mu \gamma P \\
& +\operatorname{Tr}\left\{\mathbf{\Sigma} \mathbf{G}^{2}\right\} .
\end{aligned}
$$

Similar to the solution approach for problem (17), at optimality of problem (28), one has $\mu^{\star}=\frac{K}{P}$, and

$$
\begin{aligned}
\mathbf{W} & =\zeta\left((1-\delta) \hat{\mathbf{H}}^{H} \boldsymbol{\Sigma} \mathbf{G}^{2} \hat{\mathbf{H}}+\frac{\delta \operatorname{Tr}\left\{\boldsymbol{\Sigma} \mathbf{G}^{2}\right\}}{M} \mathbf{I}+\mu^{\star} \mathbf{I}\right)^{-1} \hat{\mathbf{H}}^{H} \boldsymbol{\Sigma} \mathbf{G}^{2} \\
& =\frac{\zeta}{1-\delta} \hat{\mathbf{H}}^{H}\left(\hat{\mathbf{H}} \hat{\mathbf{H}}^{H}+\frac{\delta P \operatorname{Tr}\left\{\boldsymbol{\Sigma} \mathbf{G}^{2}\right\}+K M}{P M(1-\delta)} \boldsymbol{\Sigma}^{-1} \mathbf{G}^{-2}\right)^{-1} .
\end{aligned}
$$

Due to the normalizing step to meet the power constraint, scaling $\mathbf{W}$ by any positive factor does not affect the final normalized beamforming matrix $\tilde{\mathbf{W}}$. Thus, the optimal robust WMMSE precoder is given by

$$
\mathbf{W}^{\star}=\hat{\mathbf{H}}^{H}\left(\hat{\mathbf{H}} \hat{\mathbf{H}}^{H}+\frac{\delta \operatorname{Tr}\{\mathbf{S N R}\}+K}{M(1-\delta)} \mathbf{S N R}^{-1}\right)^{-1}
$$

where SNR $=\operatorname{diag}\left\{\mathrm{SNR}_{1}, \ldots, \mathrm{SNR}_{K}\right\}$. Finally, the optimal normalization factor $\eta^{\star}$ is given by $\eta^{\star}=$ $\sqrt{P / \operatorname{Tr}\left\{\mathbf{W}^{\star}\left(\mathbf{W}^{\star}\right)^{H}\right\}}$ and the optimal normalized beamforming matrix is $\tilde{\mathbf{W}}^{\star}=\eta^{\star} \mathbf{W}^{\star}$. Hereafter, the precoder (37) will be referred to as the Robust WMMSE precoder.

Remark 2. For the case of unquantized CDI and CQI feedbacks, one has $\tilde{\mathbf{H}}=\hat{\mathbf{H}}, \delta=0$, and $\mathbf{S N R}=(P / M) \mathbf{G}^{2} \boldsymbol{\Sigma}$. It is easy to verify that the robust WMMSE precoder (37) is exactly the same as the original WMMSE precoder (22). This observation allows us to validate the proposed robust WMMSE precoder.

Remark 3. In solving problem (28), we first assume that the effective channel gains $\mathbf{G}^{2} \boldsymbol{\Sigma}$ are known. Interestingly, the optimal solution given in (37) shows its dependence on the instantaneous SNRs $(P / M) g_{i}^{2} \rho_{i}^{\prime}$ 's at the UEs, but not on $\mathbf{G}$ or $\boldsymbol{\Sigma}$ individually. Clearly, the SNRs at the UEs can be easily estimated at the eNB by the CQI feedbacks. With unquantized CQI feedbacks, the eNB can apply the 
perfectly estimated SNRs into the precoder as in (37). In contrast, with quantized CQI feedbacks, the imperfectly estimated $\widehat{\mathrm{SNR}}_{i}$ 's can be utilized at the eNB instead.

Remark 4. With the non-weighted MSE cost function defined in problem (8), the robust MMSE design can be obtained from the optimization

$$
\begin{aligned}
& \underset{\tilde{\mathbf{W}}, \eta}{\operatorname{minimize}} \mathbb{E}\left\{\left\|\mathbf{u}-\eta^{-1} \boldsymbol{\Sigma}^{-\frac{1}{2}} \mathbf{y}\right\|^{2} \mid \hat{\mathbf{H}}\right\} \\
& \text { subject to } \operatorname{Tr}\left\{\tilde{\mathbf{W}} \tilde{\mathbf{W}}^{H}\right\} \leq P .
\end{aligned}
$$

For the case of homogeneous SNR conditions, i.e., $\rho_{i}=$ $1, \forall i$, with $M=K$, the optimal robust MMSE precoder, studied in [11], is given by (cf. Equation 28 in [11])

$$
\tilde{\mathbf{W}}=\eta \hat{\mathbf{H}}^{H}\left(\hat{\mathbf{H}} \hat{\mathbf{H}}^{H}+\frac{1+P \delta}{P(1-\delta)} \mathbf{I}\right)^{-1} .
$$

For the case of non-homogeneity under consideration, by following the similar procedure in solving problem (28), the optimal solution to problem (38) can be derived as

$$
\tilde{\mathbf{W}}=\eta \hat{\mathbf{H}}^{H} \mathbf{G}\left[\mathbf{G} \hat{\mathbf{H}} \hat{\mathbf{H}}^{H} \mathbf{G}+\left(\frac{\delta \operatorname{Tr}\left\{\mathbf{G}^{2}\right\}}{M(1-\delta)}+\frac{\operatorname{Tr}\left\{\boldsymbol{\Sigma}^{-1}\right\}}{P(1-\delta)}\right) \mathbf{I}\right]^{-1} .
$$

Although the precoder (40) seems to be different to the original precoder given in [11], one can validate the precoder (40) through the following observation. If one applies, the precoder (40) for the case of homogeneous SNR conditions by replacing $\mathbf{G}$ with its expectation as $\sqrt{M} \mathbf{I}$, and $\boldsymbol{\Sigma}$ with $\mathbf{I}$, the precoder (40) is the same as the original precoder (39). On the other hand, for the case of perfect CDI feedbacks, i.e., $\tilde{\mathbf{H}}=\hat{\mathbf{H}}$ (which makes $\mathbf{H}=\mathbf{G} \hat{\mathbf{H}}$ ) and $\delta=0$, the precoder (40) returns to original non-robust precoder (9).

It is observed that the precoder (40) requires a perfect knowledge of the channel gains in $\mathbf{G}$ and average SNRs in $\boldsymbol{\Sigma}$ individually. Thus, the precoder (40) does not fit into the CQI feedback model (24), unlike our proposed robust WMMSE precoder (37). Nevertheless, we will present some numerical simulations and compare the performances obtained by our proposed WMMSE precoder (37) and the precoder (40) (assuming $\mathbf{G}$ and $\boldsymbol{\Sigma}$ known at the eNB to implement the precoder (40)).

\section{Simulations results}

This section presents the simulation results to illustrate the achievable sum-rate of a MU-MISO system obtained from our proposed WMMSE precoding schemes, compared to well-known precoding strategies in literature (ZF-EPS, ZF-WF, RZF, DPC). In order to generate a MU system with non-homogeneous SNR conditions, we consider a two-cell system, as displayed in Figure 1. The objective is to maximize the sum-rate of the UEs in the first cell, where these UEs encounter different levels of background noise and inter-cell interference induced from the second eNB. It is assumed that the UEs are randomly located within the limit of the first cell. Thus, depending on their relative distances to the first $\mathrm{eNB}$ and the second eNB, the UEs exhibit different levels of channel gain and background noise, which lead to different SNRs. The simulation parameters, based on the LTE-Advanced physical layer architecture [17], are summarized in Table 1. The transmit power at eNB-2 is fixed at $10 \mathrm{~W}(10 \mathrm{dBW})$. In all simulations, once the eNB acquires the CSI from the UEs, it is assumed that the channel remains unchanged

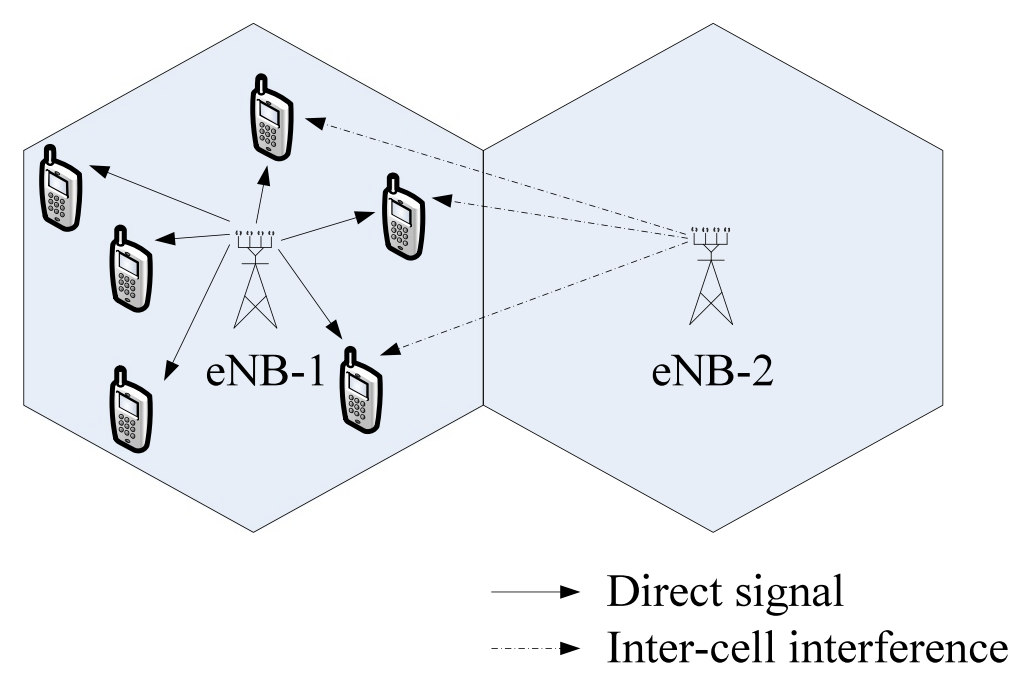

Figure $1 \mathrm{~A}$ two-cell scenario under consideration. 
Table 1 Simulation parameters and settings

\begin{tabular}{lc}
\hline Parameter & Value \\
\hline Number of cells & 2 \\
Site-to-site distance & $500 \mathrm{~m}$ \\
Antenna height - base station & $30 \mathrm{~m}$ \\
Antenna height - mobile station & $1.5 \mathrm{~m}$ \\
Carrier frequency $f_{C}$ & $2 \mathrm{GHz}$ \\
Bandwidth per radio block & $15 \mathrm{KHz}$ \\
Number of radio blocks & 180 \\
AWGN & $-174 \mathrm{dBm} / \mathrm{Hz}$ \\
eNB-2 transmit power & $10 \mathrm{dBW}$ \\
Shadowing (small-scale fading) & $0 \mathrm{~dB}$ \\
Path loss model (large-scale fading) & COST231 [18] \\
\hline
\end{tabular}

during the training and transmission phases until the next CSI update, i.e., we assume the CSI updating interval is appropriately selected such that UE mobility and resulting Doppler spread do not cause significant change in CSI before the next update of CSI.

\subsection{Achievable sum-rate with perfect CSI and average SNR}

We first examine the achievable network sum-rates when perfect CSI and average SNRs are available at the eNB. Figures 2 and 3 display the achievable network sum-rates when the network is fully loaded with the number of UEs $K$ equal to the number of transmit antennas $M$. Figure 2 plots the achievable sum-rates as a function of the number of transmit antennas $M$. The transmit power $P$ of eNB-1 is set at $10 \mathrm{dBW}$. From Figure 2, we can see a significant improvement in the achievable throughput obtained by the proposed WMMSE precoder over the ZF-EPS, ZFWF, RZF schemes, especially at high $M$ (about $10 \mathrm{~b} / \mathrm{s} / \mathrm{Hz}$ at $M=10$ ). Moreover, the performance of the proposed WMMSE precoder is very close to that of the IWMMSE scheme for all values of $M$, where the latter scheme is capable of achieving a local optimum to the sum-rate maximization (4).

In Figure 3, we plot the network sum-rate as a function of $P$ with four transmit antennas and four UEs. As the transmit power increases while the average powers of the intercell interference and background noise are unchanged, the average received SINR at each UE is expected to increase. Thus, an increase in the network sum-rate is observed for each precoding scheme. Similar to the previous simulation, the proposed WMMSE precoder also outperforms the ZF-EPS, ZF-WF, and RZF schemes while obtaining a very close performance to the locally optimal IWMMSE scheme. It is worth mentioning that the proposed WMMSE precoder is much simpler to compute than the IWMMSE scheme.

Figure 4 illustrates the evolution of the network sumrate obtained from each iteration of the IWMMSE algorithm. In this simulation, a random channel realization (with $M=K=10$ ) was chosen and $P$ is set at $10 \mathrm{dBW}$. From a random starting point of $\tilde{\mathbf{W}}$, the IWMMSE algorithm may take more than $10 \mathrm{~s}$ of iterations to converge. In contrast, the proposed WMMSE precoder

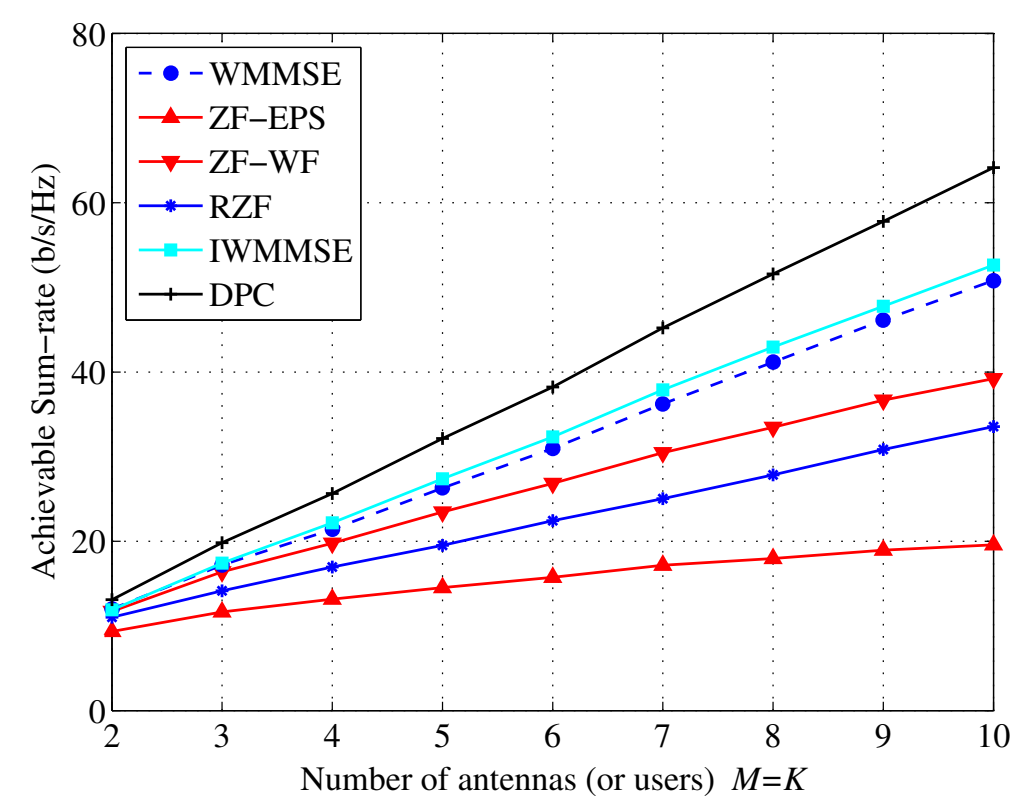

Figure 2 Network sum-rates versus $M$ (with $M=K, P=10 \mathrm{dBW}$, and perfect CSI and average SNR knowledge). 


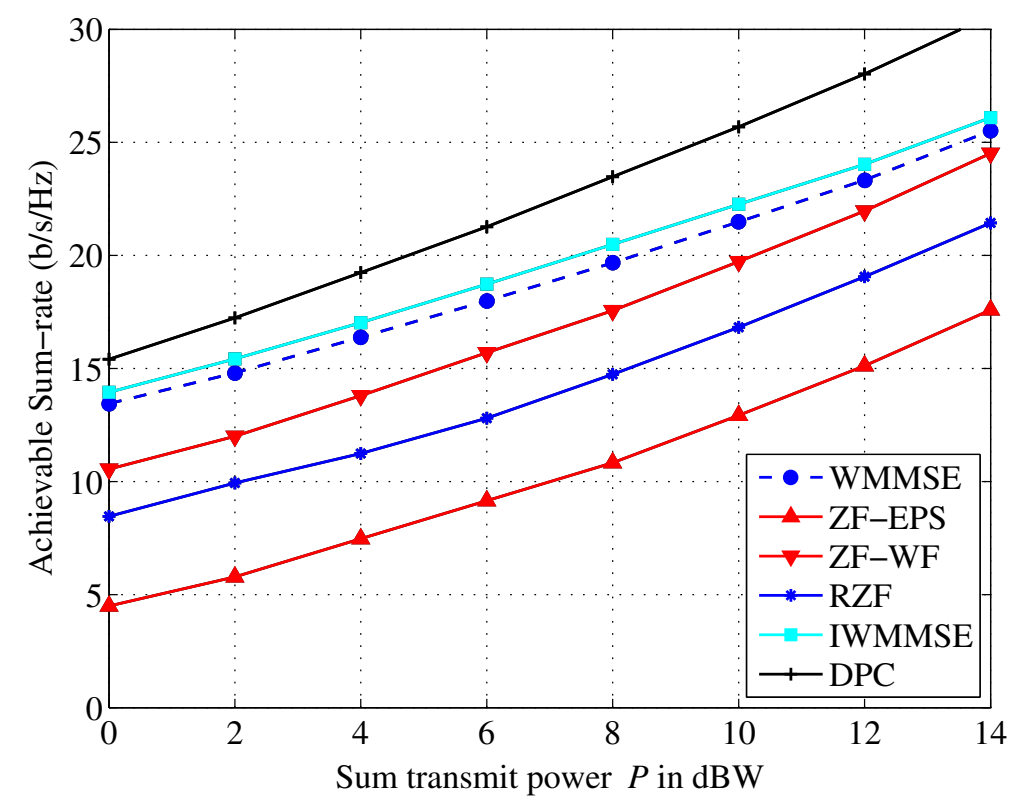

Figure 3 Network sum-rates versus $P$ (with $M=K=4$, and perfect CSI and average SNR knowledge).

(22) is directly computed without the need of any iterative procedure. At worst, the computational cost of the WMMSE precoder is equivalent to one iteration in the IWMMSE algorithm. Clearly, Figure 4 shows the foremost advantage of the proposed WMMSE precoder over the IWMMSE algorithm in terms of computational complexity. Interestingly, the proposed WMMSE precoder can serve as a starting point for the IWMMSE algorithm to speed up its convergence, as being shown in the figure.

\subsection{Achievable sum-rate with limited CDI and CQI feedbacks}

These simulations are to present the achievable network sum-rates with quantized CQI and CDI feedbacks, where we compare performance of the proposed Robust WMMSE precoder and non-robust precoders such as ZFEPS, ZF-WF, RZF, IWMMSE, DPC, and the proposed WMMSE. For CQI feedbacks, we use the CQI feedback model (24) with the 4-bit SNR-CQI mapping [15]. For CDI

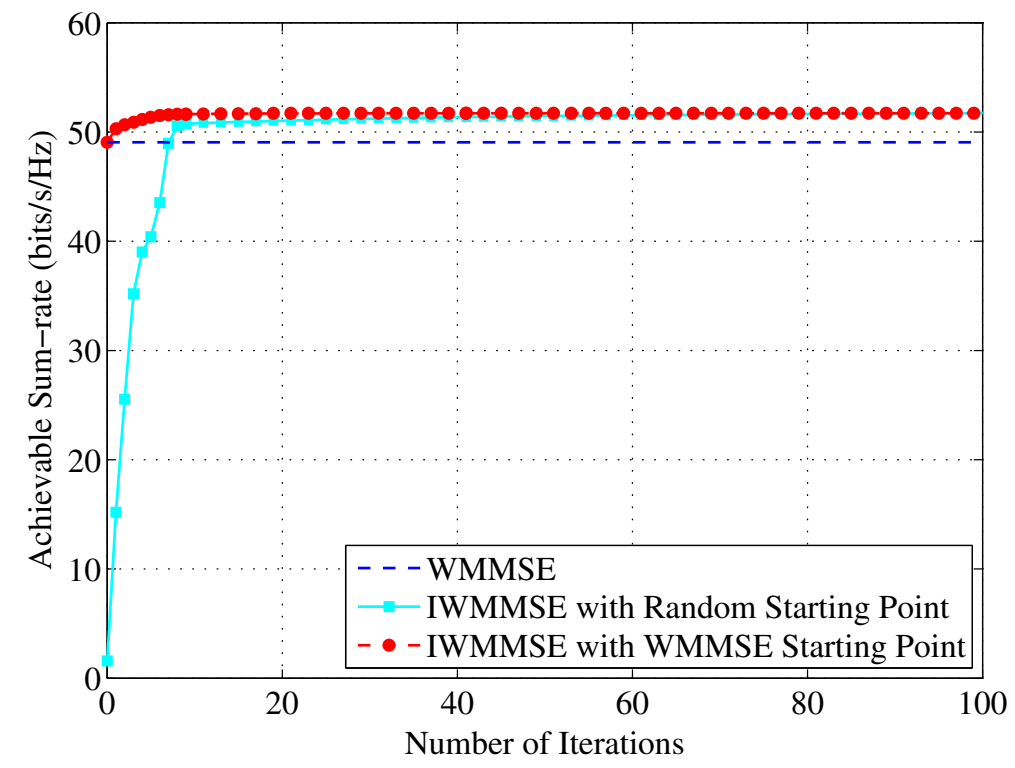

Figure 4 Convergence of IWMMSE algorithm with random starting point and starting point obtained from proposed WMMSE precoder. 


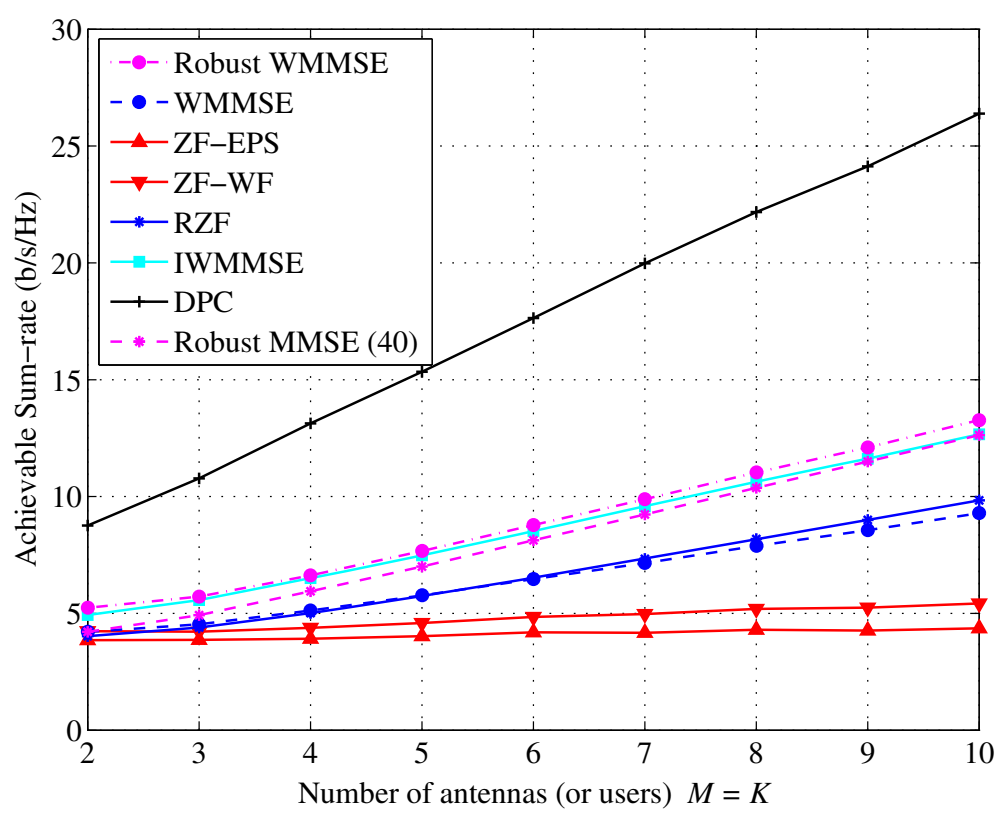

Figure 5 Network sum-rates versus $M$ (with $M=K, P=10 \mathrm{dBW}$, and quantized CQI and CDI feedbacks, $B=2(M-1)$ ).

feedbacks, the Gaussian codebook is assumed for each UE. It is stated in [16] that the number of CDI feedback bits per UE has to increase linearly with the SNR (in $\mathrm{dB}$ ) at the rate $B \approx \frac{M-1}{3} P_{\mathrm{dB}}$ in order to obtain the full multiplexing gain of $M$. Note that the result given in [16] is for systems with homogeneous SNR conditions $\left(\rho_{1}=\ldots=\right.$ $\left.\rho_{K}\right)$. Thus, with the non-homogeneous system under consideration, we also set the number of CDI feedback bits growing linearly to the number of transmit antennas $M$ or the transmit power $P$ (in $\mathrm{dBW}$ ).

Figure 5 illustrates the achievable sum-rates as a function of $M$ with $K=M$. The transmit power $P$ at eNB-1 is set to $10 \mathrm{dBW}$. To obtain the simulation result in Figure 5, we let $B=2(M-1)$. As observed from the figure, the proposed Robust WMMSE precoder significantly outperforms other non-robust linear precoders, such as ZF-EPS,

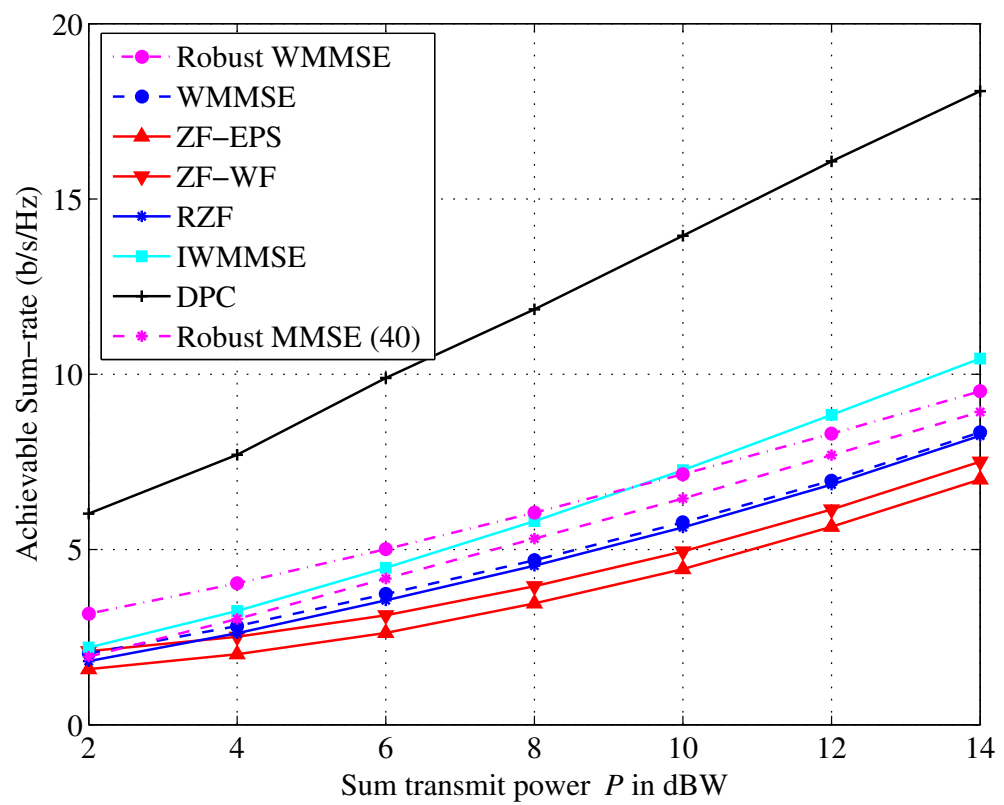

Figure 6 Network sum-rates versus $P$ (with $M=K=4$ and quantized CQI and CDI feedbacks, $B$ is set to grow equally with $P$ ). 
ZF-WF, RZF, and WMMSE, while slightly outperforming the IWMMSE scheme. When comparing to the robust MMSE precoder proposed in [11] (assuming that $\mathbf{G}$ and $\boldsymbol{\Sigma}$ are known at the eNB to implement the precoder (40)), the performance of our proposed Robust WMMSE precoder (37) is considerably better than that of the precoder (40).

Figure 6 displays the achievable network sum-rate as a function of $P$ with $M=K=4$. The number of CDI feedback bits per user $B$ is set to grow equally with $P$. For instance, if $P=10 \mathrm{dBW}, B$ is set at $10 \mathrm{bits}$. Similar to the previous simulation given in Figure 5, the proposed Robust WMMSE precoder outperforms all other non-iterative precoders, i.e, ZF-EPS, ZF-WF, RZF, and WMMSE. The proposed Robust WMMSE precoder also offers a better performance than the robust MMSE precoder (40), which was designed purposely for a homogeneous system. It is also deduced from the figure that the performance of the proposed Robust WMMSE precoder is very close to and even better than that of the non-robust precoder obtained from the IWMMSE algorithm at low- $P$ region.

\section{Conclusion}

In this work, we proposed two WMMSE precoders for a multiuser system with non-homogeneous SNR conditions. The first precoder was proposed for the system with perfect CSI and average SNRs at the eNB. Whereas the second one was proposed for the system with quantized CDI and CQI feedbacks. The principle of the proposed WMMSE precoders is to utilize the different SNR conditions to distinctly predetermine the weights and receive coefficients to the MSE cost function. Both precoders are presented in closed-form solutions, thus significantly reduce the complexity in computing them. Simulations showed that the proposed precoders significantly outperformed other well-known linear precoders, e.g., ZF-EPS, ZF-WF, RZF, while achieving a close sum-rate performance to the locally optimal IWMMSE algorithm.

\section{Endnotes}

${ }^{a}$ As suggested in [8], the conventional MSE $\mathbb{E}\left\{\left|u_{i}-\eta^{-1} y_{i}\right|^{2}\right\}$, is modified to reflect the impact of different SNR conditions at the UEs.

${ }^{\mathrm{b}}$ The instantaneous SNR defined by (24) should not be confused with the SINR defined by (3). We assume that each UE can perfectly estimate its SNR in the absence of the intra-cell interference during the training phase.

\section{Competing interests}

The authors declare that they have no competing interests.

\section{Acknowledgements}

The work presented in this paper is partly supported by the Natural Sciences and Engineering Research Council of Canada (NSERC) Discovery Program and the NSERC Collaborative Research and Development Grant with InterDigital Canada.
Received: 6 February 2014 Accepted: 22 May 2014

Published: 6 June 2014

\section{References}

1. M Costa, Writing on dirty paper. IEEE Trans. Inform. Theory 29(3), 439-441 (1983)

2. G Caire, S Shamai, On the achievable throughput of a multiantenna Gaussian broadcast channel. IEEE Trans. Inform. Theory 49(7), 1691-1706 (2003)

3. S Vishwanath, N Jindal, A Goldsmith, Duality, achievable rates, and sum-rate capacity of Gaussian MIMO, broadcast channels. IEEE Trans. Inform. Theory 49(10), 2658-2668 (2003)

4. W Yu, JM Cioffi, Sum capacity of Gaussian vector broadcast channels. IEEE Trans. Inform. Theory 50(9), 1875-1892 (2004)

5. SS Christensen, R Argawal, E de Carvalho, JM Cioffi, Weighted sum-rate maximization using weighted MMSE for MIMO-BC beamforming design. IEEE Trans. Wireless Commun. 7(12), 4792-4799 (2008)

6. CB Peel, BM Hochwald, AL Swindlehurst, A vector-perturbation technique for near-capacity multiantenna multiuser communications - Part I: channel inversion and regularization. IEEE Trans. Commun. 53(1), 195-202 (2005)

7. M Joham, K Kusume, MH Gzara, W Utschick, Transmit Wiener filter for the downlink of TDD DS-CDMA systems, in IEEE 7th Symposium on Spread-Spectrum Techniques and Applications (ISSSTA) (Prague, Czech Republic, 2-5 Sept 2002), pp. 9-13

8. H Lee, I Sohn, D Kim, KB Lee, Generalized MMSE beamforming for downlink MIMO systems, in Proceedings of IEEE International Conference on Communications (ICC 2011) (Kyoto, Japan, 5-9 June 2011), pp. 1-6

9. S Shi, M Schubert, $\mathrm{H}$ Boche, Rate optimization for multiuser MIMO systems with linear processing. IEEE Trans. Signal Process. 24(8), 4020-4030 (2008)

10. Z Wang, W Chen, Regularized zero-forcing for multiantenna broadcast channels with user selection. IEEE Wireless Commun. Lett. 1(2), 129-132 (2012)

11. C Zhang, W Xu, M Chen, Robust MMSE beamforming for multiuser MISO systems with limited feedback. IEEE Signal Proc. Lett. 16(7), 588-591 (2009)

12. KB Petersen, MS Pedersen, The Matrix Cookbook. Available: http://www2. imm.dtu.dk/pubdb/p.php?3274 (2012). Accessed 01 May 2014

13. S Boyd, L Vandenberghe, Convex Optimization. (Cambridge University Press, UK, 2004)

14. 4G Americas, 4G Mobile Broadband Evolution: 3GPP Release 10 and Beyond-HSPA+, SAE/LTE and LTE-Advanced, White Paper. (4G Americas, Bellevue, 2011)

15. CMehlführer, M Wrulich, JC Ikuno, D Bosanska, M Rupp, Simulating the long term evolution physical layer, in Proceedings of the 17th European Signal Processing Conference (EUSIPCO 2009) (Glasgow, Scotland, 24), pp. $1471-1478$

16. N Jindal, MIMO broadcast channels with finite-rate feedback. IEEE Trans. Inform. Theory. 52(11), 4045-5050 (2006)

17. 3GPP, Further advancements for E-UTRA physical layer aspects. 3GPP, Tech. Rep. TR 36.814 V9.0.0 (2010)

18. A Goldsmith, Wireless Communications. (Cambridge University Press, UK, 2004)

doi:10.1186/1687-6180-2014-85

Cite this article as: Nguyen and Le-Ngoc: MMSE precoding for multiuser MISO downlink transmission with non-homogeneous user SNR conditions. EURASIP Journal on Advances in Signal Processing 2014 2014:85. 